\title{
ANÁLISIS DE LA REFORMA EDUCATIVA ESPAÑOLA DE 2013. ¿QUÉ SE ENTIENDE POR CALIDAD Y MEJORA?
}

\author{
Patricia Gómez HERNÁNDEZ ${ }^{1}$ \\ Carlos Monge LÓPEZ ${ }^{2}$ \\ Eladio Sebastián HEREDERO ${ }^{3}$
}

RESUMEN: La educación, a lo largo de la historia, ha evolucionado para adaptarse a las necesidades de cada momento. Unos de los cambios más significativos en este aspecto son los que hacen referencia a las reformas de las leyes orgánicas que la regulan. Éstos, junto con las temáticas de investigación en torno a la calidad y la mejora educativa, son aspectos relevantes en la actualidad. Por tanto, en esta investigación se plantea un estudio de la última reforma educativa en relación a los términos destacados. Para ello se desarrolla un análisis documental de las leyes implicadas (la Ley Orgánica 2/2006, de 3 de mayo, de Educación y la Ley Orgánica 8/2013, de 9 de diciembre, para la Mejora de la Calidad Educativa), utilizando el análisis de contenido como técnica para la recogida y análisis de los datos, en el que los resultados generales indican que la nueva ley añade, modifica y suprime artículos de la ley anterior en torno a estas palabras clave. En conclusión, ambas leyes protagonistas en el estudio no entienden del mismo modo la calidad ni la mejora.

PALABRAS CLAVE: Calidad. Cambio. Mejora. Reforma.

\section{Introducción}

La educación en España ha progresado a lo largo de la historia pasando por diferentes momentos. Desde sus inicios, cuando algunos niños y niñas iban a casa del profesor a aprender nociones básicas, ha evolucionado hasta conformarse como es en la actualidad, donde gran parte es de carácter obligatorio y existen multitud enseñanzas especializadas. Sin embargo, los cambios más notables que ha sufrido la educación vienen dados por las leyes y reformas que la han regulado en cada momento (Esquema 1), conceptualizando de este modo la reforma como las "modificaciones estructurales del sistema educativo como respuestas a cambios que se consideran necesarios." (GAIRÍN, 2009, p.22).

\footnotetext{
${ }^{1}$ Doctoranda en Educación. UAH - Universidad de Alcalá. Facultad de Educación. Guadalajara - España. 19001 - patricia.gomezh@mail.uah.es

${ }^{2}$ UAH - Universidad de Alcalá. Facultad de Educación - Departamento de Ciencias de la Educación. Guadalajara - España. 19001 - carlos.monge@ mail.uah.es

${ }^{3}$ UAH - Universidad de Alcalá. Escuela Universitaria de Magisterio - Departamento de Didáctica. Guadalajara - Espanha. 19001 - eladio.sebastian@uah.es
} 


\section{Esquema 1 - Diferentes Leyes Orgánicas de Educación en España}

\section{Ley Moyano}

Ley de Instrucción Pública de 9 de septiembre de 1856

$$
\text { LGE }
$$

Ley 14/1970, de 4 de agosto, General de Educación y Financiamiento de la Reforma Educativa

$$
\text { LODE }
$$

Ley Orgánica 8/1985, de 3 de julio, reguladora del Derecho a la Educación

\section{LOGSE}

Ley Orgánica 1/1990, de 3 de octubre, de Ordenación General del Sistema Educativo

\section{LOPEG}

Ley Orgánica 9/1995, de 20 de noviembre, de la Participación, la Evaluación y el Gobierno de los centros docentes

$$
\text { LOCE }
$$

Ley Orgánica 10/2002, de 23 de diciembre, de Calidad de la Educación

$$
\text { LOE }
$$

Ley Orgánica 2/2006, de 3 de mayo, de Educación

\section{LOMCE}

Ley Orgánica 8/2013, de 9 de diciembre, para la Mejora de la Calidad Educativa

Fuente: Elaboración propia.

Algunas leyes han durado más que otras $\mathrm{y}$ han tenido mayor impacto $\mathrm{y}$ trascendencia en la historia de la educación, como por ejemplo la Ley 14/1970, de 4 de agosto, General de Educación y Financiamiento de la Reforma Educativa, que supuso una verdadera revolución en el ámbito educativo. Por el contrario, otras, como la Ley Orgánica 9/1995, de 20 de noviembre, de la Participación, la Evaluación y el Gobierno de los centros docentes, ni siquiera se han llevado a la práctica sino que han quedado en papel mojado.

En este sentido, la literatura científica recoge multitud de estudios que ofrecen una panorámica general de las políticas educativas a lo largo de la historia de un país, como es el caso de Colombia (MARTÍN; GARCÍA, 2014), Portugal (AFONSO, 1998) o España (BELTRÁN; HERNÁNDEZ; MONTANÉ, 2008). Del mismo modo, se pueden encontrar investigaciones que comparan las políticas educativas estatales entre diferentes países, por ejemplo entre España y Suecia (GARCÍA, 2014) o España y 
Brasil (SEBASTIAN, 2011). En esta línea, también destacan estudios en torno al análisis legislativo desde diferentes Comunidades Autónomas (ARGIBAY et al., 2011; JIMÉNEZ, 2003; SEBASTIAN, 2000).

Las temáticas de investigación educativa han variado, al igual que las leyes, según los intereses y necesidades del momento. Un ejemplo de ello es el caso de la calidad educativa, cuyo auge se encuentra ya reflejado en la Ley Orgánica 1/1990, de 3 de octubre de 1990, de Ordenación General del Sistema Educativo, siendo su principal objetivo la consecución de una educación de calidad (PÉREZ et al. 2004). Además, en la Ley Orgánica 10/2002, de 23 de diciembre, de Calidad de la Educación, este término presenta unas connotaciones mayores y apreciables desde el primer momento, quedando reflejadas en el título que la enmarca. A partir de entonces se está potenciando este concepto en los documentos educativos, sobre todo en los de carácter prescriptivo, como es el caso de la introducción de dicho vocablo en el título de la última ley educativa aprobada (Ley Orgánica, 8/2013, de 9 de diciembre, para la Mejora de la Calidad Educativa), y esto repercute tanto en las políticas educativas como en las tendencias de investigación en el ámbito educativo actuales.

A tales efectos, se han dado diferentes definiciones de calidad que conllevan adheridas ciertas connotaciones dependiendo del autor y del momento en el que se plantearon. Barrett et al. (2006), tras un profundo y exhaustivo análisis de informes de gran prestigio a nivel internacional en torno a la calidad educativa, recogen cinco dimensiones del término: (a) efectividad, (b) eficiencia, (c) equidad, (d) relevancia y (e) sostenibilidad. A ello añaden que estos cinco factores pueden servir como base para analizar la calidad de los procesos de cambio planificados orientados a la mejora. En este sentido, una de las publicaciones más relevantes sobre la gestión de la calidad en educación dentro del contexto español es la presentada por López (1994), quien enumera siete indicadores de gestión de la calidad:

- Dirección-liderazgo.

- Recursos humanos: (a) selección, (b) formación, (c) reconocimiento/recompensas y (d) plan de carrera.

- Participación.

- Autonomía.

- Orientación.

- Impacto en la sociedad.

- Resultados. 
Así, muchas definiciones que plantean diferentes autores en torno a la calidad están asociadas al término mejora, entendida como la incorporación de "[...] unas respuestas más aceptables que las existentes respecto a los valores o prioridades que se hayan establecido." (GAIRÍN, 2009, p.22). Del mismo modo, se pueden encontrar estudios relevantes al respecto, como el que presentan Brighouse y Woods (2001) distinguiendo cinco tipos de variables que inciden enormemente sobre la mejora de los centros educativos: (a) dirección, (b) revisión colectiva, (c) entornos de aprendizaje, (d) formación del profesorado y (e) participación de las familias y la comunidad.

Por todo ello, y partiendo de las concepciones y experiencias descritas, el principal objetivo que se plantea en esta investigación es analizar el contenido de la última reforma del sistema educativo español, es decir el paso de la Ley Orgánica 2/2006, de 3 de mayo, de Educación a la Ley Orgánica 8/2013, de 9 de diciembre, para la Mejora de la Calidad Educativa, en torno a dos términos clave: mejora y calidad.

\section{Método}

El diseño metodológico consiste en el análisis documental utilizando el análisis de contenido como estrategia para la recogida y análisis de datos (COHEN; MANION; MORRISON, 2007). Concretamente, se trata de un análisis de contenido exploratorio, cuantitativo-cualitativo, primario, longitudinal y semántico, siguiendo un procedimiento secuenciado (GARCÍA, 2003):

1. Universo de contenidos y selección de la muestra. En este caso, y procurando seguir los criterios de exhaustividad, representatividad, homogeneidad y pertinencia, los textos analizados se corresponden con la última reforma del sistema educativo español: (a) la Ley Orgánica 2/2006, de 3 de mayo de Educación y (b) la Ley Orgánica 8/2013, de 9 de diciembre, para la Mejora de la Calidad Educativa.

2. Unidades de análisis. Los términos mejora y calidad (y sus respectivos sinónimos) se corresponden con estas unidades, destacando dos componentes esenciales: las unidades de base gramatical y el análisis de las palabras.

3. Reglas de enumeración. Aquí se contempla fundamentalmente la frecuencia y la frecuencia ponderada, y por ende también la presencia-ausencia.

4. Establecimiento de las categorías.

5. Interpretación de los resultados. 
En este estudio la fiabilidad se identifica con la coincidencia entre investigadores durante la enumeración de las unidades de análisis. Y la validez viene dada por el juicio de expertos en mejora de la calidad educativa.

\section{Resultados y discusión}

La actual reforma educativa se focaliza principalmente sobre el cambio de la Ley Orgánica 2/2006, de 3 de mayo, de Educación a la Ley Orgánica 8/2013, de 9 de diciembre, para la Mejora de la Calidad Educativa. Sin embargo, también este último documento modifica parcialmente otras legislaciones, como la Ley Orgánica 6/2001, de 21 de diciembre, de Universidades.

En términos de calidad y mejora, la Ley Orgánica 2/2006, de 3 de mayo, de Educación contempla hasta 45 veces el vocablo calidad y 41 el de mejora, siguiendo una tendencia muy similar la Ley Orgánica 8/2013, de 9 de diciembre, para la Mejora de la Calidad Educativa, con 50 y 48 veces respectivamente. Esto no quiere decir que una ley posea más calidad y pretensiones de mejora que otra, sino que intenta destacar unos términos sobre otros, sin afán o con él de lograrlos, lo que se puede apreciar ya en el título de esta última ley.

En el cuerpo del texto de la nueva ley la palabra mejora aparece exactamente el doble de veces menos que en el preámbulo, mientras que en la ley antigua sigue una proporción que ronda prácticamente el 50\%. En este sentido, parece que la Ley Orgánica 8/2013, de 9 de diciembre, para la Mejora de la Calidad en Educación no interpreta de la misma manera que la Ley Orgánica 2/2006, de 3 de mayo, de Educación la necesidad de una puesta en práctica basada en una justificación previa. Y con respecto al término calidad, en el caso de la Ley Orgánica 2/2006, de 3 de mayo, de Educación se sigue la misma tendencia homogénea, mientras que en el caso de la otra ley la diferencia se reduce considerablemente.

La Ley Orgánica 8/2013, de 9 de diciembre, para la Mejora de la Calidad Educativa modifica la ley anterior en términos explícitos de calidad y mejora, bien sea por medio de la adición, eliminación o sustitución de artículos. En el primer caso, se añaden en numerosas ocasiones dichos términos, como en el Artículo 111 bis. Cabe destacar también el añadido del Artículo 122 bis, pues señala hasta 16 veces el término calidad, lo que hace que un solo artículo eleve considerablemente las frecuencias analizadas, en detrimento de la Ley Orgánica 2/2006, de 3 de mayo, de Educación. 
1. [...] Estas medidas también irán encaminadas a potenciar y a facilitar el aprovechamiento de los registros administrativos en el marco de las estadísticas educativas estatales, para posibilitar la ampliación de la información estadística referida al alumnado, el profesorado, los centros y las gestiones educativas, lo que redundará en la mejora de las herramientas de análisis y de seguimiento de la actividad educativa y de las medidas de mejora de la calidad del Sistema Educativo Español. (ESPAÑA, 2013).

En cuanto al procedimiento de sustitución, la nueva ley recurre constantemente a cambiar artículos de la Ley Orgánica 2/2006, de 3 de mayo, de Educación por unos nuevos. En el caso que repercute a los términos calidad y mejora, puede señalarse como llamativo el Artículo 127, pues a pesar de su modificación posee el mismo número de palabras referentes a calidad y mejora antes y después del cambio. También puede ejemplificarse una sustitución mediante el Artículo 143 en torno a estas palabras clave, pues modifica un artículo donde en un principio no aparecen estos dos conceptos generando uno nuevo con 2 frecuencias de cada uno.

Precisamente, el Artículo 143 se centra sobre la evaluación general del sistema educativo, donde la nueva ley enfatiza la estandarización y evaluación externa de los centros educativos partiendo de su más profundo ideario pedagógico. De hecho, la Ley Orgánica 8/2013, de 9 de diciembre, para la Mejora de la Calidad Educativa contempla en el Preámbulo que esa mejora de la calidad no puede entenderse sin los datos aportados por las pruebas de evaluación internacional como PISA (Programme for International Student Assessment).

1. El Instituto Nacional de Evaluación Educativa, en colaboración con las Administraciones educativas, elaborará planes plurianuales de evaluación general del sistema educativo. Previamente a su realización se harán públicos los criterios y procedimientos de evaluación. Asimismo, el Instituto Nacional de Evaluación Educativa establecerá los estándares metodológicos y científicos que garanticen la calidad, validez y fiabilidad de las evaluaciones educativas, en colaboración con las Administraciones educativas. [...]

4. Con el fin de posibilitar el diagnóstico de debilidades y el diseño e implantación de medidas de mejora de la calidad del Sistema Educativo Español, el Ministerio de Educación, Cultura y Deporte, en colaboración con las Administraciones educativas, arbitrará los mecanismos para posibilitar la incorporación de información adicional al tratamiento estadístico conjunto, que permita un mejor análisis de los factores que afectan al 
rendimiento educativo y la comparación basada en el valor añadido. (ESPAÑA, 2013).

También se dan supresiones de ciertos artículos, pero sin variar sustancialmente las frecuencias de los términos mejora y calidad. Este mismo hecho sucede con la modificación de la Ley Orgánica 6/2001, de 21 de diciembre, de Universidades, pues la nueva legislación educativa únicamente sustituye el Artículo 42, así como con la modificación de la Ley Orgánica 8/1985, de 3 de julio, reguladora del Derecho a la Educación. En este sentido, hasta el momento, parece que la nueva legislación española en materia educativa no pretende resaltar los términos calidad y mejora en el sistema universitario.

Finalmente, a pesar de que la Ley Orgánica 2/2006, de 3 de mayo, de Educación y la Ley Orgánica 8/2013, de 9 de diciembre, para la Mejora de la Calidad Educativa son dos documentos que en diseño e ideario educativo difieren considerablemente, parece sorprendente que ambas sigan los mismos principios explícitos de calidad y mejora, recogidos en el Artículo 1, pues aunque se modifican y añaden párrafos en dicho artículo, ninguno de los nuevos contempla los términos calidad y mejora.

\section{Conclusiones}

Efectivamente, las reformas en educación tienen como principal objetivo introducir cambios orientados a la mejora de la calidad, como aspecto necesario para la adaptación del sistema educativo a las características cambiantes de la sociedad. A pesar de esa necesidad, también es lógica la pretensión hacia una cierta estabilidad que garantice y conforme unas bases legislativas en torno a un ideario pedagógico común y en relación a los deberes y derechos humanos.

A modo de conclusión, parece evidente que tanto la Ley Orgánica 2/2006, de 3 de mayo, de Educación como la Ley Orgánica 8/2013, de 9 de diciembre, para la Mejora de la Calidad Educativa destacan los términos calidad y mejora dentro del texto, si bien es cierto que la primera incide más en la justificación/preámbulo.

Ambas leyes, a pesar de incidir en la calidad y mejora de la educación, parece que no entienden de la misma manera estos dos términos, puesto que cada una diseña el desarrollo de actuaciones distintas.

Como principal limitación de este estudio se puede destacar que en primera instancia se ha seguido un análisis de contenido clásico, donde sólo se han analizado los documentos originales referentes a ambas leyes. No obstante, y para resaltar otros 
aspectos, posteriormente se ha realizado un análisis de corte más cualitativo que lo complementa. En este sentido, como prospectivas de futuro, puede señalarse el análisis en torno a la mejora de la calidad de la reforma en la práctica real de aula.

\section{ANALYSIS OF EDUCATION SPANISH REFORM IN 2013: IMPROVEMENT AND QUALITY EDUCATION CONCEPT.}

ABSTRACT: Education, along history, has evolved to suit itself needs of the moment. Ones of these more significant changes in these terms are around reforms of organic laws that regulate the education. These aspects, together with research topics about educational quality and improvement, are relevant elements today. Therefore, in this study, a documental analysis of involved educational laws (Organic Law 2/2006, May 3rd, of Education and Organic Law 8/2013, December 9th, for Educational Quality Improvement) is planned around these highlighted terms, using content analysis as data collect and analysis technique, where generated results indicate that the new law adds, modifies and deletes some articles of previous law around these two keywords. In conclusion, both laws do not understand equally the quality neither the improvement.

KEYWORDS: Change. Improvement. Quality. Reform.

\section{REFERENCIAS}

AFONSO, A. J. Políticas educativas e avaliação educacional: para uma análise sociológica da reforma educativa em Portugal: (1985-1995). Braga: Universidade do Minho, 1998.

ARGIBAY, M. et al. Informe sobre la situación de ocho comunidades autónomas. Bilbao: Universidad del País Vasco, 2011.

BARRETT, A. M. et al. The concept of quality in education: a review of the "international" literature on the concept of quality in education. Bristol: University of Bristol, 2006.

BELTRÁN, J.; HERNÁNDEZ, F.; MONTANÉ, A. Tradición y modernidad en las políticas educativas en España: una revisión de las últimas décadas. Revista Iberoamericana de Educación, Madrid, v.48, pp. 53-71, 2008.

BRIGHOUSE, T.; WOODS, D. Cómo mejorar los centros docentes. Madrid: La Muralla, 2001.

COHEN, L.; MANION, L.; MORRISON, K. Research methods in education. 6.ed. Nueva York: Routledge, 2007. 
ESPAÑA. Ley Orgánica 8/2013, de 9 de diciembre de 2013. Para la Mejora de la Calidad Educativa. Boletín Oficial del Estado, Madrid, n.295, p.97858-97921, 10 dic. 2013.

Ley Orgánica 2/2006, de 3 de mayo de 2006. Educación. Boletín Oficial del Estado, Madrid, n.106, p.17158-17207, 4 mayo 2006.

. Ley Orgánica 10/2002, de 23 de diciembre de 2002. Calidad de la Educación. Boletín Oficial del Estado, Madrid, n.307, p.45188-45220, 24 dic. 2002.

Ley Orgánica 6/2001, de 21 de diciembre de 2001. Universidades. Boletín Oficial del Estado, Madrid, n.307, p.49400-49425, 24 dic. 2001.

Ley Orgánica 9/1995, de 20 de noviembre de 1995. La Participación, la Evaluación y el Gobierno de los centros docentes. Boletín Oficial del Estado, Madrid, n.278, p.33651-33665, 21 nov. 1995.

. Ley Orgánica 1/1990, de 3 de octubre de 1990. Ordenación General del Sistema Educativo. Boletín Oficial del Estado, Madrid, n.238, p.28927-28942, 4 oct. 1990.

Ley Orgánica 8/1985, de 3 de julio de 1985. Reguladora del Derecho a la Educación. Boletín Oficial del Estado, Madrid, n.159, p.21015-21022, 4 jul. 1985.

Ley 14/1970, de 4 de agosto de 1970. General de Educación y Financiamiento de la Reforma Educativa. Boletín Oficial del Estado, Madrid, n.187, p.12525-125466, ago. 1970.

GAIRÍN, J. Cambio y mejora: la innovación en el aula, equipo de profesores y centro. In: PAREDES, J.; HERRÁN, A. de la. (Coord.). La práctica de la innovación educativa. Madrid: Síntesis, 2009. p. 21-48.

GARCÍA, J. L. Métodos de investigación en educación. Madrid: Universidad Nacional de Educación a Distancia, 2003. v.2.

GARCÍA, P. Convergencias y divergencias entre el sistema educativo sueco y el español. Revista Española de Educación Comparada, [S.1.], n.23, p.203-222, 2014.

JIMÉNEZ, J. La educación en las comunidades autónomas. Cuadernos de Pedagogía, Barcelona, n.323, p.45-75, 2003.

LÓPEZ, F. La gestión de la calidad en educación. Madrid: La Muralla, 1994.

MARTÍN, M. A.; GARCÍA, W. Análisis comparado de las políticas educativas en Colombia: escuela nueva vs. educación indígena. Revista Española de Educación Comparada, [S.1.], n.23, p.223-240, 2004.

PÉREZ, R. et al. Hacia una educación de calidad: gestión, instrumentos y evaluación. Madrid: Ediciones Narcea, 2004. 
SEBASTIAN, E. Estrategias organizativas y curriculares para una escuela inclusiva: análisis de la situación en España y Brasil: estudio de casos. In: SEBASTIÁN, E. Y.; MARTIN, M. (Coord.). Transferencia del conocimiento a partir de prácticas educativas en los contextos español y brasileño. Alcalá de Henares: Editorial Servicio de Publicaciones de la UAH, 2011. p.33-64.

Adaptaciones curriculares en las comunidades autónomas. Revista Escuela Española, Madrid, n.3439, p.22-23, 2000. 